\title{
Changes in haematological parameters and oxidative stress response of goats subjected to road transport stress in a hot humid tropical environment
}

\begin{abstract}
Even though studies investigating the effect of road transportation on haematological parameters in goats from temperate and hot tropical environments are numerous, few studies have focused on the recovery rate following transportation, especially in the hot, humid tropical environment. This study investigated the haematological parameters and oxidative stress response of goats subjected to $7 \mathrm{~h}$ of road transportation in the hot, humid tropical environment and the possible recovery period. Thirty-five healthy Boer goats, aged $2 \mathrm{i} 3$, weighing $20 \ddot{\mathrm{I}} 25 \mathrm{~kg}$, were divided into two groups, designated A and B, of 30 and 5 animals, respectively. Group A was transported for $7 \mathrm{~h}$, and blood samples were collected before, 3.5 h on transit, after transport, and on days 3, 7, 16 and 26 post-transport, while five goats served as control. Plasma and haemolysate were prepared and used to assay malondialdehyde, superoxide dismutase and glutathione alongside haematological parameters. The differential leukocyte counts of group A were altered following transportation as neutrophils, monocytes and neutrophil/lymphocyte ratios increased significantly $(\mathrm{P} \mathbb{A}[\mathrm{C} .01)$ during transport compared to group B. Malondialdehyde value increased significantly $(\mathrm{P} \mathbb{W}[\mathbf{0} .01)$ following transportation through day 3 post-transport, while superoxide dismutase and glutathione activities decreased simultaneously following transportation and increased from day 3 through day 7 post-transportation. This study revealed that goats subjected to $7 \mathrm{~h}$ of transportation in the hot humid tropical condition experience haematological derangements and oxidative stress which take an average period of 3 to 16 days for recovery.
\end{abstract}

Keyword: Boer goats; Malondialdehyde; Oxidative stress response; Road transportation; Superoxide dismutase 